\title{
Darboux transformation of the general Hirota equation: multisoliton solutions, breather solutions, and rogue wave solutions
}

\author{
Deng-Shan Wang*, Fei Chen and Xiao-Yong Wen
}

\author{
*Correspondence: \\ wangdsh1980@163.com \\ School of Applied Science, Beijing \\ Information Science and \\ Technology University, Beijing, \\ 100192, China
}

\begin{abstract}
In this paper, we investigate the exact solutions and conservation laws of a general Hirota equation. Firstly, the $\mathrm{N}$-fold Darboux transformation of this equation is proposed. Then by choosing three kinds of seed solutions, the multisoliton solutions, breather solutions, and rogue wave solutions of the general Hirota equation are obtained based on the Darboux transformation. Finally, the conservation laws of this equation are derived by using its linear spectral problem. The results in this paper may be useful in the study of ultrashort optical solitons in optical fibers.
\end{abstract}

Keywords: Darboux transformation; multisoliton solutions; breather solutions; rogue wave solutions; optical fibers

\section{Introduction}

During the past decades, many researches have been focused on optical solitons due to their potential applications in optical fiber long-distance transmission systems. According to the theoretical report and experimental results in [1,2], optical solitons are based on the balance between the group velocity dispersion and self-phase modulation in the picosecond regime, and the propagation of such a soliton is governed by the standard nonlinear Schrödinger equation (NLS) [3, 4],

$$
i u_{t}+\frac{1}{2} u_{x x}+|u|^{2} u=0
$$

where $u=u(x, t)$ denotes the slowly varying complex envelope of the wave, and subscripts $x$ and $t$ are the longitudinal distance and retarded time, respectively.

In recent years, the ultrashort pulses have attracted much interest because of their applications in optical fibers. When they are considered, the NLS equation (1) cannot describe the corresponding physical characteristics, and it accounts for the following three points: first, the fourth-order dispersion should be considered when the pulse width is below 10 femtoseconds $[5,6]$; second, the higher-order nonlinearities should not be neglected when the optical field frequency approaches a resonant frequency of the optical fibers material [6,7]; third, the self-steepening and self-frequency shift should be included when extremely narrow pulse has very high optical intensity as the fourth-order dispersion

(c) 2016 Wang et al. This article is distributed under the terms of the Creative Commons Attribution 4.0 International License (http://creativecommons.org/licenses/by/4.0/), which permits unrestricted use, distribution, and reproduction in any medium, provided you give appropriate credit to the original author(s) and the source, provide a link to the Creative Commons license, and indicate if changes were made. 
and cubic-quintic nonlinearities are considered [4, 8]. Comprehensively considering these three factors, in this paper, we study the higher-order nonlinear Schrödinger equation

$$
u_{t}=i \alpha_{1} u_{x x}+i \alpha_{2}|u|^{2} u+\alpha_{3} \epsilon u_{x x x}+3 \alpha_{4} \epsilon|u|^{2} u_{x}+2 \alpha_{5} \epsilon|u|^{2} u_{x}
$$

where $u$ is the complex amplitude of the pulse envelope, the parameter $\epsilon$ denotes the relative width of the spectrum that arises due to the quasi-monochromaticity, and $\alpha_{1}, \alpha_{2}$, $\alpha_{3}, \alpha_{4}$, and $\alpha_{5}$ are real constants. It is shown in [9] that when $\alpha_{2}=2 \delta^{2} \alpha_{3}, \alpha_{4}=6 \delta^{2} \alpha_{3}$, and $\alpha_{5}=-6 \delta^{2} \alpha_{3}$, Eq. (2) becomes the bright soliton version of the general Hirota equation

$$
u_{t}=\alpha_{1}\left(i u_{x x}+2 i \delta^{2}|u|^{2} u\right)+\epsilon \alpha_{3}\left(u_{x x x}+6 \delta^{2}|u|^{2} u_{x}\right)
$$

which further becomes the following general Hirota equation by putting $\alpha_{1}=\alpha$ and $\alpha_{3}=1$ :

$$
u_{t}=\alpha\left(i u_{x x}+2 i \delta^{2}|u|^{2} u\right)+\epsilon\left(u_{x x x}+6 \delta^{2}|u|^{2} u_{x}\right) .
$$

Note that when $\epsilon=0, \alpha=\frac{1}{2}$, and $\delta^{2}=1$, the general Hirota equation (4) becomes the standard focusing NLS equation (1). When $\alpha=0$, it becomes the complex modified Korteweg-de Vries equation [10], namely

$$
u_{t}=\epsilon\left(u_{x x x}+6 \delta^{2}|u|^{2} u_{x}\right)
$$

The aim of this paper is to find some new-type explicitly exact solutions and conservation laws of the general Hirota equation (4). In recent years, some work [11-18] has been done to investigate the exact solutions and numerical solutions of various differential equation. Especially, some results on the Hirota equation are proposed by analytical [11-14] and numerical [18] methods. However, here we consider the general Hirota equation (4) in a different way and derive the multisoliton solutions, breather solutions, rogue wave solutions, and conservation laws of this equation. This paper is organized as follows. The Lax pair and $N$-fold Darboux transformation of Eq. (4) are presented in Section 2. In Section 3, the multisoliton solutions, breather solutions, and rogue wave solutions are obtained via the proposed Darboux transformation, and the dynamics of these exact solutions is analyzed by their density distributions. In Section 4, some conservation laws for the Eq. (4) are listed explicitly. Conclusions are addressed in the last section.

\section{Lax pair and Darboux transformation}

The Lax pair (i.e., linear spectral problem) [9] of the general Hirota equation (4) is

$$
\varphi_{x}=U \varphi, \quad \varphi_{t}=V \varphi,
$$

with matrices $U$ and $V$ of the forms

$$
U=-i \lambda U_{0}+U_{1}, \quad V=i \lambda^{3} V_{3}-\lambda^{2} V_{2}+\lambda V_{1}+V_{0},
$$


where $\varphi=(f, g)^{T}$ (here $T$ denotes the transpose), $\lambda$ is a spectral parameter, and

$$
\begin{aligned}
U_{0} & =\left(\begin{array}{cc}
1 & 0 \\
0 & -1
\end{array}\right), \quad U_{1}=\left(\begin{array}{cc}
0 & \delta u \\
-\delta u^{*} & 0
\end{array}\right), \\
V_{3} & =4 \epsilon U_{0}, \quad V_{2}=2 i \alpha U_{0}+4 \epsilon U_{1}, \\
V_{1} & =\left(\begin{array}{cc}
-2 i \epsilon \delta^{2}|u|^{2} & 2\left(\alpha \delta u-i \epsilon \delta u_{x}\right) \\
-2\left(\alpha \delta u^{*}-i \epsilon \delta u_{x}^{*}\right) & 2 i \epsilon \delta^{2}|u|^{2}
\end{array}\right), \quad V_{0}=\left(\begin{array}{cc}
a_{11} & a_{12} \\
a_{21} & -a_{11}
\end{array}\right),
\end{aligned}
$$

with

$$
\begin{aligned}
& a_{11}=\epsilon \delta^{2} u_{x} u^{*}-\epsilon \delta^{2} u_{x}^{*} u+i \alpha \delta^{2}|u|^{2}, \quad a_{12}=\epsilon \delta u_{x x}+i \alpha \delta u_{x}+2 \epsilon \delta^{3}|u|^{2} u \\
& a_{21}=-\epsilon \delta u_{x x}^{*}+i \alpha \delta u_{x}^{*}-2 \epsilon \delta^{3}|u|^{2} u^{*} .
\end{aligned}
$$

Through direct calculations, we can verify that the zero-curvature equation $U_{t}-V_{x}+$ $[U, V]=0$ exactly gives rise to the general Hirota equation (4).

Darboux transformation (DT) technique is a method that can derive multisoliton solutions from trivial seed solutions in a purely algebraic procedure for the integrable nonlinear wave equations [19]. Main feature of DT is that the Lax pair associated with the nonlinear wave equations remains covariant under the gauge transformation. By using the Lax pair (6) we construct the gauge transformation of Eq. (4) as

$$
\varphi^{(1)}=T^{(1)} \varphi=(\lambda I-S) \varphi,
$$

where $I$ denotes the identity matrix, and $S=\left(s_{i j}^{(0)}\right)_{2 \times 2}$ is a $2 \times 2$ matrix. Furthermore, if the matrix $S$ takes the form

$$
S=H \Lambda H^{-1}
$$

with

$$
\begin{aligned}
& \Lambda=\left(\begin{array}{cc}
\lambda_{1} & 0 \\
0 & \lambda_{1}^{*}
\end{array}\right), \quad H=\left(\begin{array}{cc}
f_{1} & -g_{1}^{*} \\
g_{1} & f_{1}^{*}
\end{array}\right), \\
& S=\frac{1}{\Delta}\left(\begin{array}{cc}
\lambda_{1} f_{1} f_{1}^{*}+\lambda_{1}^{*} g_{1} g_{1}^{*} & f_{1} g_{1}^{*}\left(\lambda_{1}-\lambda_{1}^{*}\right) \\
f_{1}^{*} g_{1}\left(\lambda_{1}-\lambda_{1}^{*}\right) & \lambda_{1}^{*} f_{1} f_{1}^{*}+\lambda_{1} g_{1} g_{1}^{*}
\end{array}\right), \\
& \operatorname{det} H=\Delta=\left|f_{1}\right|^{2}+\left|g_{1}\right|^{2},
\end{aligned}
$$

then we can verify that if $\left(f_{1}, g_{1}\right)^{T}$ is a solution of the Lax pair (6) with $\lambda=\lambda_{1}$, then $\left(-g_{1}^{*}, f_{1}^{*}\right)^{T}$ is also a solution of the Lax pair (6) corresponding to $\lambda=\lambda_{1}^{*}$. Thus, under the gauge transformation $\varphi^{(1)}=T^{(1)} \varphi$, the Lax pair (6) becomes

$$
\varphi_{x}^{(1)}=U^{(1)} \varphi^{(1)}, \quad \varphi_{t}^{(1)}=V^{(1)} \varphi^{(1)},
$$

with

$$
U^{(1)}=-i \lambda U_{0}^{(1)}+U_{1}^{(1)}, \quad V^{(1)}=i \lambda^{3} V_{3}^{(1)}-\lambda^{2} V_{2}^{(1)}+\lambda V_{1}^{(1)}+V_{0}^{(1)}
$$


where $U_{0}^{(1)}, U_{1}^{(1)}$, and $V_{i}^{(1)}(j=0,1,2,3)$ have the same forms as $U_{0}, U_{1}$, and $V_{i}(j=0,1,2,3)$ in Eqs. (8)-(11) with $u$ replaced by $u^{(1)}$.

Through the gauge transformation (12), the relationships among $T^{(1)}, U$, and $U^{(1)}$ can be found:

$$
\begin{aligned}
& T_{x}^{(1)}+T^{(1)} U=U^{(1)} T^{(1)}, \\
& T_{t}^{(1)}+T^{(1)} V=V^{(1)} T^{(1)} .
\end{aligned}
$$

By direct computation based on Eqs. (19)-(20), we can obtain a relation between potential functions $u^{(1)}$ and $u$ :

$$
u^{(1)}=u-\frac{2 i s_{21}^{(0)}}{\delta} .
$$

So the DT of the general Hirota equation (4) is defined as

$$
\varphi^{(1)}=T^{(1)} \varphi, \quad u^{(1)}=u-\frac{2 i s_{21}^{(0)}}{\delta},
$$

where

$$
T^{(1)}=\lambda_{1} I-S=\left(\begin{array}{cc}
\lambda_{1}-s_{11}^{(0)} & -s_{12}^{(0)} \\
-s_{21}^{(0)} & \lambda_{1}-s_{22}^{(0)}
\end{array}\right), \quad s_{21}^{(0)}=\frac{f_{1}^{*} g_{1}\left(\lambda_{1}-\lambda_{1}^{*}\right)}{\left|f_{1}\right|^{2}+\left|g_{1}\right|^{2}} .
$$

If $N$ distinct seed solutions $\varphi_{k}(k=1,2, \ldots, N)$ of Eq. (6) are given, then the basic DT may be iterated. To do the second step of transformation, we employ $\varphi_{2}$, which is mapped to $\varphi_{2}^{(1)}=\left.T^{(1)}\right|_{\lambda=\lambda_{2}} \varphi_{2}$; therefore,

$$
\varphi^{(2)}=T^{(2)} \varphi^{(1)}=T^{(2)} T^{(1)} \varphi, \quad u^{(2)}=u^{(1)}-\frac{2 i s_{21}^{(1)}}{\delta}=u-\frac{2 i}{\delta}\left(s_{21}^{(0)}+s_{21}^{(1)}\right),
$$

where

$$
T^{(2)}=\left(\begin{array}{cc}
\lambda_{2}-s_{11}^{(1)} & -s_{12}^{(1)} \\
-s_{21}^{(1)} & \lambda_{2}-s_{22}^{(1)}
\end{array}\right), \quad s_{21}^{(1)}=\frac{f_{2}^{(1) *} g_{2}^{(1)}\left(\lambda_{2}-\lambda_{2}^{*}\right)}{\left|f_{2}^{(1)}\right|^{2}+\left|g_{2}^{(1)}\right|^{2}} .
$$

In the general case, we have the following theorem.

Theorem 1 Let $\varphi_{1}, \varphi_{2}, \ldots, \varphi_{N}$ be $N$ distinct solutions of the spectral problem (6) at $\lambda_{1}, \ldots, \lambda_{N}$, respectively. Then the $N$-fold DT for the general Hirota equation (4) is

$$
\varphi^{(n)}=T^{(n)} T^{(n-1)} \cdots T^{(1)} \varphi, \quad u^{(n)}=u-\frac{2 i}{\delta} \sum_{j=0}^{n-1} s_{21}^{(j)}
$$

with

$$
T^{(j+1)}=\left(\begin{array}{cc}
\lambda_{j+1}-s_{11}^{(j)} & -s_{12}^{(j)} \\
-s_{21}^{(j)} & \lambda_{j+1}-s_{22}^{(j)}
\end{array}\right), \quad s_{21}^{(j)}=\frac{f_{j+1}^{(j) *} g_{j+1}^{(j)}\left(\lambda_{j+1}-\lambda_{j+1}^{*}\right)}{\left|f_{j+1}^{(j)}\right|^{2}+\left|g_{j+1}^{(j)}\right|^{2}},
$$




$$
\varphi^{(j-1)}=\left.\left(T^{(j-1)} T^{(j-2)} \cdots T^{(1)}\right)\right|_{\lambda=\lambda_{j}} \varphi,
$$

where $\varphi^{(j+1)}=\left(f_{j+1}^{j}, g_{j+1}^{j}\right)^{T}$ is the solution of the spectral problem (6) at $\lambda=\lambda_{j+1}$.

\section{Multisoliton solutions, breather solutions, and rogue wave solutions}

In this section, we give some explicit solutions of Eq. (4) via the DT in Section 2. Now we take the nonzero continuous wave (cw) solution $u=c e^{i(a x+b t)}$ as the initial seed for Eq. (4), where $a, b, c$ are all real parameters. Equation (4) requires the frequency $b$ to satisfy the following nonlinear dispersion relation:

$$
b=\epsilon\left(6 a \delta^{2} c^{2}-a^{3}\right)+\alpha\left(2 \delta^{2} c^{2}-a^{2}\right) .
$$

Thus, the cw seed solution of the general Hirota equation (4) is

$$
u=c e^{i\left(a x+\left(\epsilon\left(6 a \delta^{2} c^{2}-a^{3}\right)+\alpha\left(2 \delta^{2} c^{2}-a^{2}\right)\right) t\right)} .
$$

Setting $\left(f_{1}, g_{1}\right)^{T}$ to be the solution of the spectral problem (6), we have

$$
\begin{aligned}
& f_{1 x}=-i \lambda f_{1}+\delta u g_{1}, \quad g_{1 x}=i \lambda g_{1}-\delta u^{*} f_{1}, \\
& f_{1 t}=v_{11} f_{1}+v_{12} g_{1}, \quad g_{1 t}=v_{21} f_{1}+v_{22} g_{1},
\end{aligned}
$$

where

$$
\begin{aligned}
& v_{11}=4 i \epsilon \lambda^{3}-2 i \alpha \lambda^{2}-2 i \epsilon \delta^{2} u^{2} \lambda+i \alpha \delta^{2} u^{2}, \\
& v_{22}=-4 I \epsilon \lambda^{3}+2 i \alpha \lambda^{2}+2 i \epsilon \delta^{2} u^{2} \lambda-i \alpha \delta^{2} u^{2}, \\
& v_{12}=-4 \epsilon \delta u \lambda^{2}+\left(2 \alpha \delta u-2 i \epsilon \delta u_{x}\right) \lambda+\epsilon \delta u_{x x}+i \alpha \delta u_{x}+2 \epsilon \delta^{3} u^{3}, \\
& v_{21}=4 \epsilon \delta u \lambda^{2}+\left(-2 \alpha \delta u-2 i \epsilon \delta u_{x}\right) \lambda-\epsilon \delta u_{x x}+i \alpha \delta u_{x}-2 \epsilon \delta^{3} u^{3} .
\end{aligned}
$$

After tedious computations, we obtain that

$$
\begin{aligned}
& f_{1}=\left(C_{1} e^{A}+C_{2} e^{-A}\right) e^{B}, \\
& g_{1}=i\left[C_{2} \frac{\sqrt{(a+2 \lambda)^{2}+4 \delta^{2} c^{2}}+(a+2 \lambda)}{2 \delta c}-C_{1} \frac{\sqrt{(a+2 \lambda)^{2}+4 \delta^{2} c^{2}}-(a+2 \lambda)}{2 \delta c}\right] e^{-B},
\end{aligned}
$$

with

$$
\begin{aligned}
& A=\frac{i \sqrt{(a+2 \lambda)^{2}+4 \delta^{2} c^{2}}}{2}\left[x-\left(a^{2} \epsilon+4 \lambda^{2} \epsilon-2 \delta^{2} c^{2} \epsilon-2 \lambda a \epsilon+\alpha a-2 \lambda \alpha\right) t\right], \\
& B=\frac{i}{2}\left[a x-\left(a^{3} \epsilon-6 \epsilon \delta^{2} c^{2} a+a^{2} \alpha-2 \alpha \delta^{2} c^{2}\right) t\right] .
\end{aligned}
$$

\subsection{Multisoliton solutions}

In this subsection, we present the multisoliton solutions of Eq. (4) explicitly. To do so, take $c=0$, that is, zero initial seed solution for Eq. (4). The solutions of the spectral problem (6) in Eqs. (37) and (38) are reduced to the following forms:

$$
f_{1}=e^{-i \lambda x+\left(4 i \epsilon \lambda^{3}-2 i \alpha \lambda^{2}\right) t}, \quad g_{1}=e^{i \lambda x-\left(4 i \epsilon \lambda^{3}-2 i \alpha \lambda^{2}\right) t} .
$$


(a)

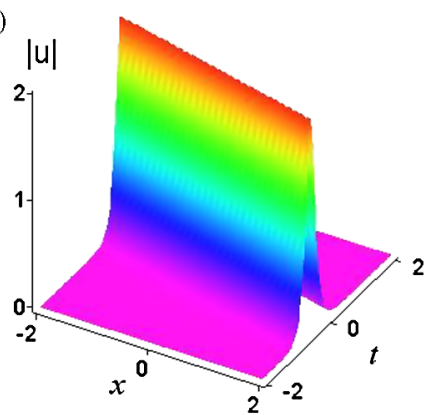

(b)

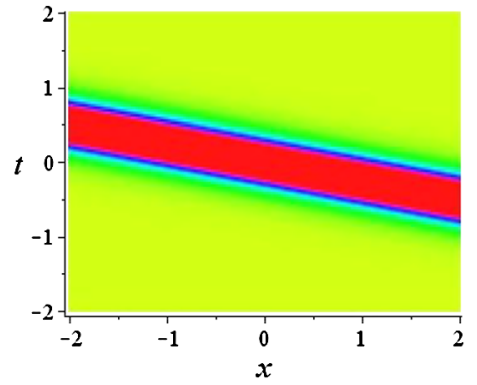

Figure 1 Profile and contour plots of one soliton. (a) Evolution of the one-soliton solution $u_{s s}^{(1)}$ via Eq. (42) with parameters $\alpha=1, \epsilon=1, \delta=1, \lambda=i$. (b) The corresponding density plot of $u_{s s}^{(1)}$ in (a).

(1) When $N=1$ in the Darboux transformation of Theorem 1, after some calculations, we have

$$
u_{s s}^{(1)}=-\frac{2 \eta_{1}}{\delta} e^{-2 i\left(\varepsilon_{1} x+t\left(4 \epsilon \varepsilon_{1}\left(3 \eta_{1}^{2}-\varepsilon_{1}^{2}\right)+2 \alpha\left(\varepsilon_{1}^{2}-\eta_{1}^{2}\right)\right)\right)} \operatorname{sech}\left(2 \eta_{1}\left(x+4\left(\epsilon \eta_{1}^{2}-3 \epsilon \varepsilon_{1}^{2}+\varepsilon_{1} \alpha\right) t\right)\right),
$$

where $\lambda=\varepsilon_{1}+i \eta_{1}$, and $u_{s s}^{(N)}$ is the $N$ th-order soliton solution.

The solution $u_{s s}^{(1)}$ represents a bright single soliton whose dynamic features are delineated in Figure 1. We can conclude some physical quantities from this bright single soliton: the maximum amplitude is $\left|\frac{2 \eta_{1}}{\delta}\right|$; the width is $\frac{1}{2 \eta_{1}}$; the envelop velocity is $-4 \eta_{1}\left(\epsilon \eta_{1}^{2}-3 \epsilon \varepsilon_{1}^{2}+\varepsilon_{1} \alpha\right)$; the frequency is $-4 \epsilon \varepsilon_{1}\left(3 \eta_{1}^{2}-\varepsilon_{1}^{2}\right)-2 \alpha\left(\varepsilon_{1}^{2}-\eta_{1}^{2}\right)$, and the energy $E$ is equal to $\left|\frac{4 \eta_{1}}{\delta^{2}}\right|$.

(2) When $N=2$ in the Darboux transformation of Theorem 1, we obtain the following two-soliton solution of Eq. (4):

$$
u_{s s}^{(2)}=u_{s s}^{(1)}-\frac{2 i}{\delta} \frac{f_{2}^{(1) *} g_{2}^{(1)}\left(\lambda_{2}-\lambda_{2}^{*}\right)}{\left|f_{2}^{(1)}\right|^{2}+\left|g_{2}^{(1)}\right|^{2}},
$$

where $u_{s s}^{(1)}$ is given in Eq. (42), and $\left(f_{2}^{(1)}, g_{2}^{(1)}\right)^{T}$ is the solution of the spectral problem (6) at $\lambda=\lambda_{2}$.

The dynamics of the two-soliton solution $u_{s s}^{(2)}$ is delineated in Figure 2 with parameters $\alpha=1, \epsilon=1, \delta=1, \lambda_{1}=i, \lambda_{2}=2 i$. The solution $u_{s s}^{(2)}$ represents the elastic interaction between two bright solitons whose dynamic features are delineated in Figure 2. Figure 2 shows the overtaking interaction between two bell-shape solitons for $u_{s s}^{(2)}$ in Eq. (43), from which we can find that the solitonic shapes and amplitudes have not changed after the interaction.

(3) When $N=3$, we obtain the following three-soliton solution of Eq. (4):

$$
u_{s s}^{(3)}=u_{s s}^{(2)}-\frac{2 i}{\delta} \frac{f_{3}^{(2) *} g_{3}^{(2)}\left(\lambda_{3}-\lambda_{3}^{*}\right)}{\left|f_{3}^{(2)}\right|^{2}+\left|g_{3}^{(2)}\right|^{2}},
$$

where $u_{s s}^{(2)}$ is given in Eq. (43), and $\left(f_{3}^{(2)}, g_{3}^{(2)}\right)^{T}$ is the solution of the spectral problem (6) at $\lambda=\lambda_{3}$.

The dynamic features of the three-soliton solution $u_{s s}^{(3)}$ is delineated in Figure 3 with parameters $\alpha=1, \epsilon=1, \delta=1, \lambda_{1}=i, \lambda_{2}=2 i, \lambda_{3}=4 i$. The solution $u_{s s}^{(3)}$ represents the elastic interaction among three bright solitons whose dynamic features are delineated in 
(a)

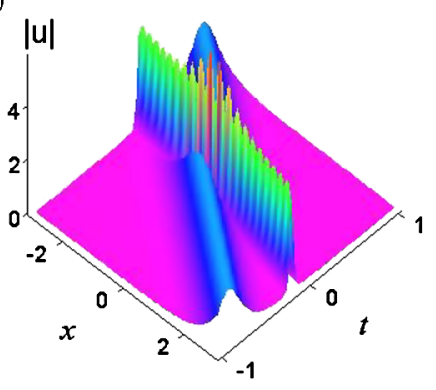

(b)

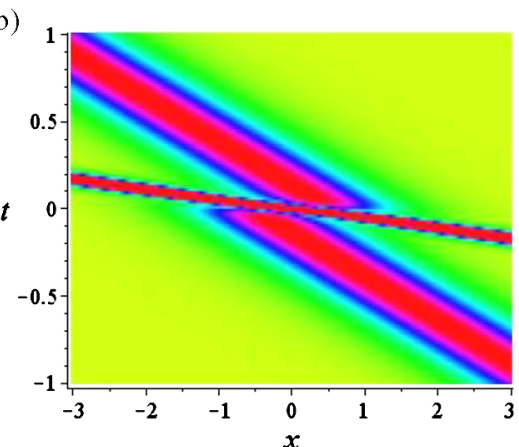

Figure 2 Profile and contour plots of two solitons. (a) Evolution of the two-soliton solution $u_{s s}^{(2)}$ represented by Eq. (43) with parameters $\alpha=1, \epsilon=1, \delta=1, \lambda_{1}=i, \lambda_{2}=2 i$. (b) The corresponding density plot of $u_{s s}^{(2)}$ in (a).

(a)

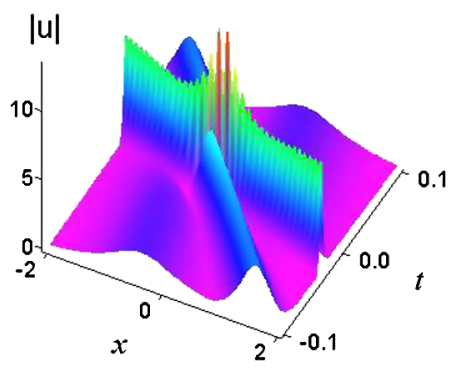

(b)

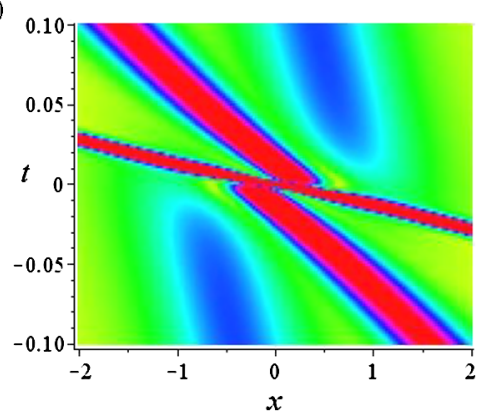

Figure 3 Profile and contour plots of three solitons. (a) Evolution of the three-soliton solution $u_{s s}^{(3)}$ via Eq. (44) with parameters $\alpha=1, \epsilon=1, \delta=1, \lambda_{1}=i, \lambda_{2}=2 i, \lambda_{3}=4 i$. (b) The corresponding density plot of $u_{s s}^{(3)}$ in (a).

Figure 3. Figure 3 shows the overtaking interaction among three bell-shape bright solitons for $u_{s s}^{(3)}$ in Eq. (44), from which we can find that the solitonic shapes and amplitudes have not changed after the interaction.

(4) When $N=4$, we also obtain the four-soliton solution of Eq. (4) by the same procedure:

$$
u_{s s}^{(4)}=u_{s s}^{(3)}-\frac{2 i}{\delta} \frac{f_{4}^{(3) *} g_{4}^{(3)}\left(\lambda_{4}-\lambda_{4}^{*}\right)}{\left|f_{4}^{(3)}\right|^{2}+\left|g_{4}^{(3)}\right|^{2}},
$$

where $u_{s s}^{(3)}$ is given in Eq. (44), and $\left(f_{4}^{(3)}, g_{4}^{(3)}\right)^{T}$ is the solution of the spectral problem (6) at $\lambda=\lambda_{4}$.

The dynamic features of the four-soliton solution $u_{s s}^{(4)}$ is delineated in Figure 4 with parameters $\alpha=1, \epsilon=1, \delta=1, \lambda_{1}=i, \lambda_{2}=2 i, \lambda_{3}=4 i, \lambda_{4}=3 i$. The solution $u_{s s}^{(4)}$ represents the elastic interaction among four bright solitons whose dynamic features are shown in Figure 4. Figure 4 shows the overtaking interaction among four bell-shape bright solitons for solution $u_{s s}^{(4)}$ in Eq. (45), from which we can find that the solitonic shapes and amplitudes have not changed after the interaction.

We remark that we can also get the high-order soliton solutions of the general Hirota equation (4) by continuing iteration of the DT in Theorem 1. 
(a)

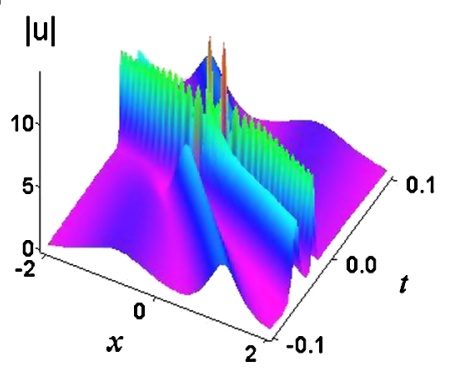

(b)

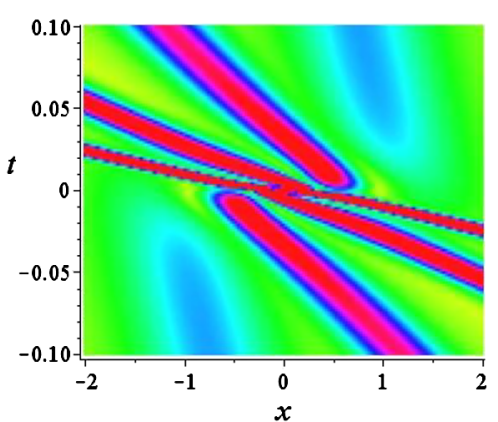

Figure 4 Profile and contour plots of four solitons. (a) Evolution of the four-soliton solution $u_{s s}^{(4)}$ via Eq. (45) with parameters $\alpha=1, \epsilon=1, \delta=1, \lambda_{1}=i, \lambda_{2}=2 i, \lambda_{3}=4 i, \lambda_{4}=3 i$. (b) The corresponding density plot of $u_{s s}^{(4)}$ in (a).

(a)

(c)
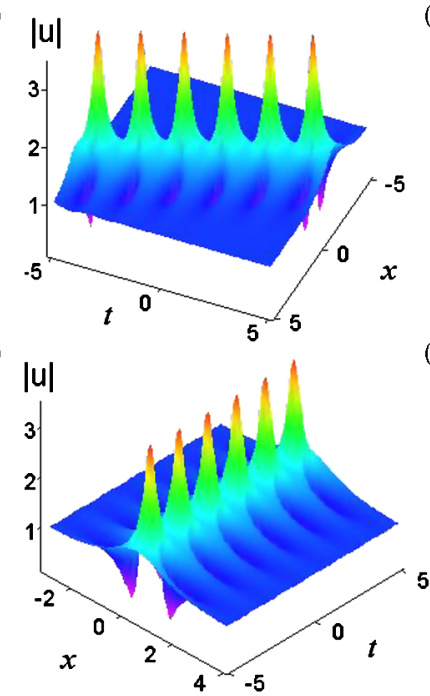

(b)

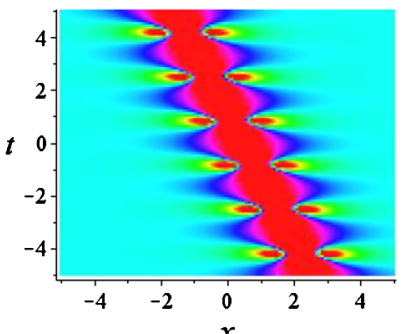

(d)

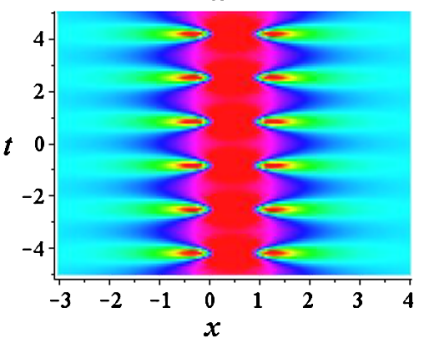

Figure 5 Profile and contour plots of one breather localized in space. (a) Evolution of the one-breather solution $u_{b s}^{(1)}$ with parameters $\alpha=1, \epsilon=0.1, \delta=1, a=0, \lambda_{1}=\frac{5}{4} i$. (b) The corresponding density plot of $u_{b s}^{(1)}$ in (a). (c) Evolution plot of $u_{b s}^{(1)}$ with parameters $\alpha=1, \epsilon=0, \delta=1, a=0, \lambda_{1}=\frac{5}{4} i$. (d) The corresponding density plot of $u_{b s}^{(1)}$ in (c).

\subsection{Multibreather solutions}

In this subsection, we consider the case of parameter $c \neq 0$ in the seed solution of the general Hirota equation (4), which we will start with the plane wave seed solution. Without loss of generality, assuming that $c=1$ and substituting the solution of the spectral problem (6) given by Eqs. (37) and (38) into the 1-fold DT in Eq. (22), the one-breather solution of the general Hirota equation (4) can be obtained immediately. Hereby, iterating the DT by using Theorem 1 we can derive the multibreather solutions of Eq. (4), where $u_{b s}^{(N)}$ represents the $N$ th-order breather solution for simplicity. In the following, we only discuss two cases, $N=1$ and $N=2$. Moreover, we omit the expressions of the breather solutions because they are too long to write down.

(1) When $N=1$, the one-breather solution $u_{b s}^{(1)}$ is derived from the plane wave initial solution and 1-fold DT in Eq. (22), whose dynamic features are delineated in Figures 5 and 6. 
(a)

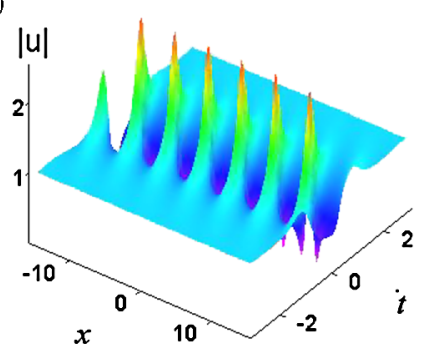

(c)

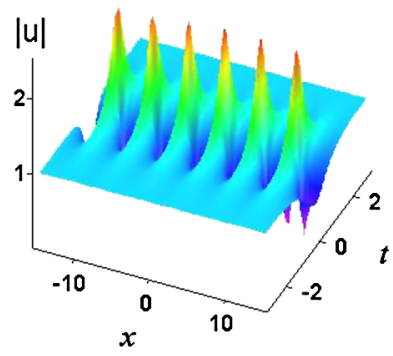

(b)

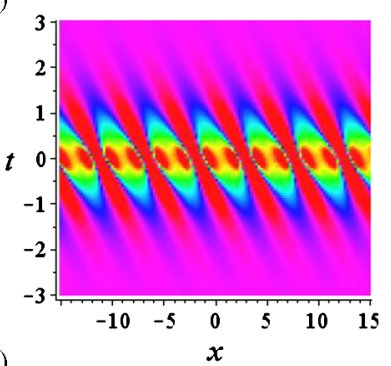

(d)

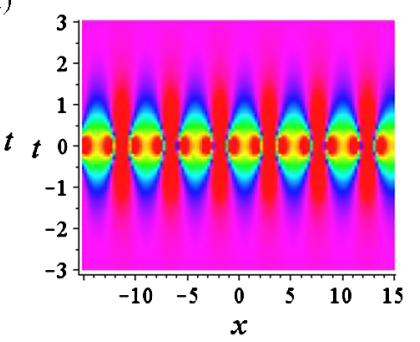

Figure 6 Profile and contour plots of one breather localized in time. (a) Evolution of the one-breather-soliton solution $u_{b s}^{(1)}$ with parameters $\alpha=1, \epsilon=0.5, \delta=1, a=0, \lambda_{1}=\frac{3}{4} i$. (b) The corresponding density plot of $u_{b s}^{(1)}$ in (a). (c) Evolution plot of $u_{b s}^{(1)}$ with parameters $\alpha=1, \epsilon=0, \delta=1, a=0, \lambda_{1}=\frac{3}{4}$. (d) The corresponding density plot of $u_{b s}^{(1)}$ in (c).

Figures 5(a), (b) and 6(a), (b) display the structures of the one-breather solution $u_{b s}^{(1)}$ for the general Hirota equation (4) with $\epsilon \neq 0$, and Figures 5(c), (d) and 6(c), (d) display the same case except the parameter $\epsilon=0$. Wes see that the one-breather solution is periodic both in space and time for the parameter $\epsilon \neq 0$. Moreover, comparing Figure 5 with Figure 6, we observe that when $|\operatorname{Im}(\lambda)|>1$, the evolution and density plots for the one-breather solution $u_{b s}^{(1)}$ are periodic in time (i.e., Ma soliton), however, they are periodic in space (i.e., Akhmediev soliton) for $0<|\operatorname{Im}(\lambda)|<1$ under the same parameters. We can see that the parameter $\epsilon$ can change the shape and the arrangement of bright solitons.

(2) When $N=2$, the two-breather solution $u_{b s}^{(2)}$ is also derived from the plane wave initial solution and 2-fold DT in Theorem 1, whose dynamic features are delineated in Figures 7 and 8 .

From Figures 7 and 8 we know that: when $\epsilon=0, \operatorname{Re}\left(\lambda_{1}\right)=0, \operatorname{Re}\left(\lambda_{2}\right)=0,0<\left|\operatorname{Im}\left(\lambda_{1}\right)\right|<1$, $\left|\operatorname{Im}\left(\lambda_{2}\right)\right|>1$, the solution $u_{b s}^{(2)}$ represents the elastic interaction between a space periodic breather solution and a time-periodic breather solution (see Figure 7(a), (b)); when $\epsilon=0$, $\operatorname{Re}\left(\lambda_{1}\right)=0, \operatorname{Re}\left(\lambda_{2}\right) \neq 0,0<\left|\operatorname{Im}\left(\lambda_{1}\right)\right|<1$, the solution $u_{b s}^{(2)}$ represents the elastic interaction between two breather solutions, in witch one is a space-periodic breather solution, the other is a periodic breather solution in time and space (see Figure 7(c), (d)); when $\epsilon=0, \operatorname{Re}\left(\lambda_{1}\right)=0, \operatorname{Re}\left(\lambda_{2}\right)=0,0<\left|\operatorname{Im}\left(\lambda_{1}\right)\right|<1,0<\left|\operatorname{Im}\left(\lambda_{2}\right)\right|<1$, the solution $u_{b s}^{(2)}$ represents the elastic interaction between two space periodic breather soliton solutions (see Figure 8(a), (b)); when $\epsilon=0, \operatorname{Re}\left(\lambda_{1}\right)=0, \operatorname{Re}\left(\lambda_{2}\right)=0,\left|\operatorname{Im}\left(\lambda_{1}\right)\right|>1,\left|\operatorname{Im}\left(\lambda_{2}\right)\right|>1$, the solution $u_{b s}^{(2)}$ represents the interaction between two time-periodic breather soliton solutions (see Figure 8(c), (d)). 
(a)

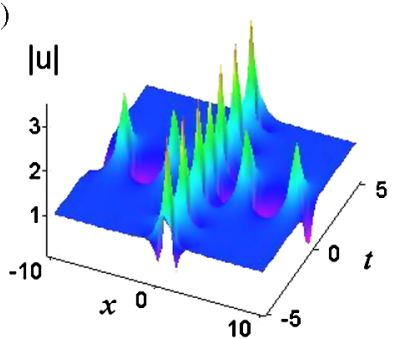

(c)

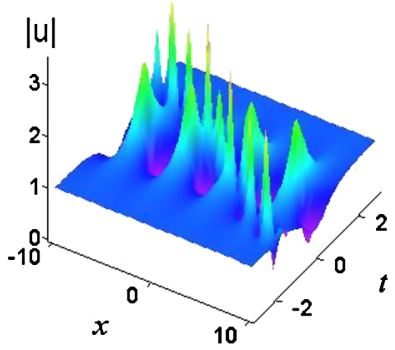

(b)

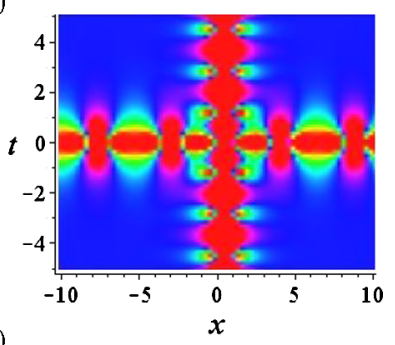

(d)

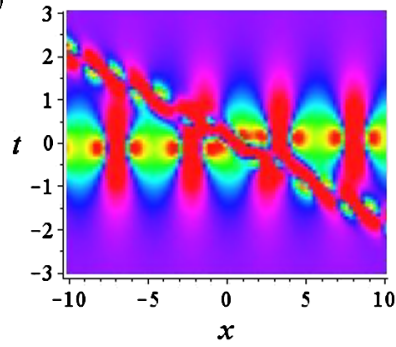

Figure 7 Profile and contour plots of two breathers of first kind. (a) Evolution of the two-breather solution $u_{b s}^{(2)}$ with parameters $\alpha=1, \epsilon=0, \delta=1, a=0, \lambda_{1}=\frac{3}{4} i_{1} \lambda_{2}=\frac{5}{4} i$. (b) The corresponding density plot of $u_{b s}^{(2)}$ in (a). (c) Evolution plot of $u_{b s}^{(2)}$ with parameters $\alpha=1, \epsilon=0, \delta=1, a=0, \lambda_{1}=\frac{3}{4} i, \lambda_{2}=1+i$. (d) The corresponding density plot of $u_{b s}^{(2)}$ in (c).

(a)

(c)
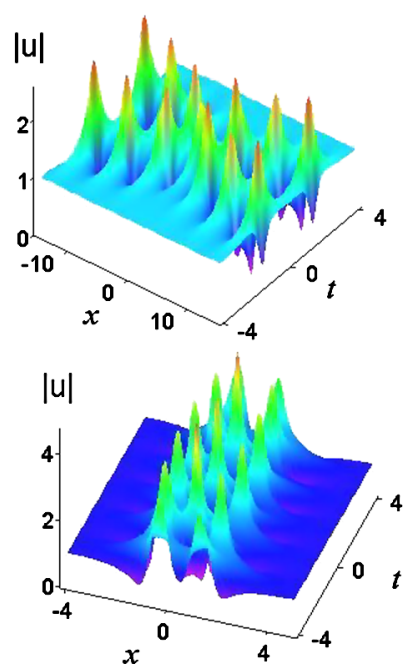

(b)

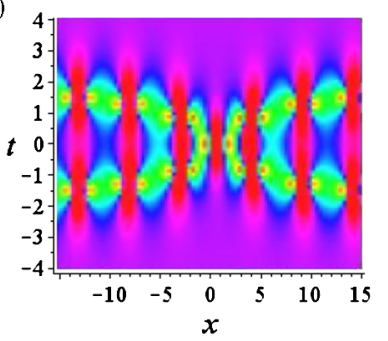

(d)

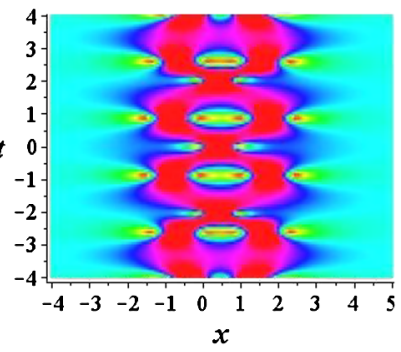

Figure 8 Profile and contour plots of two breathers of second kind. (a) Evolution of the two-breather solution $u_{b s}^{(2)}$ with parameters $\alpha=1, \epsilon=0, \delta=1, a=0, \lambda_{1}=\frac{3}{4} i, \lambda_{2}=\frac{4}{5} i$. (b) The corresponding density plot of $u_{b s}^{(2)}$ in (a). (c) Evolution of the two-breather solution $u_{b s}^{(2)}$ with parameters $\alpha=1, \epsilon=0, \delta=1, a=0, \lambda_{1}=\frac{5}{4} i$, $\lambda_{2}=\frac{3}{2} i$. (d) The corresponding density plot of $u_{b s}^{(2)}$ in (c).

\subsection{Rogue wave solutions}

Next, we manage to search a generalized DT. Suppose that $\varphi_{2}=\varphi_{1}\left(\lambda_{1}+\sigma\right)$ is a special solution for Eq. (6). Then after transformation we have $\varphi_{2}^{(1)}=T_{1}^{(1)} \varphi_{2}$. Expanding $\varphi_{2}$ at $\lambda_{1}$, we have from [20]

$$
\varphi_{1}\left(\lambda_{1}+\sigma\right)=\varphi_{1}+\varphi_{1}^{[1]} \sigma+\varphi_{1}^{[2]} \sigma^{2}+\cdots+\varphi_{1}^{[N]} \sigma^{N}+\cdots
$$


where $\varphi_{1}^{[k]}=\left.\frac{1}{k !} \frac{\partial^{k}}{\partial \lambda^{k}} \varphi_{1}(\lambda)\right|_{\lambda=\lambda_{1}}$, and $\sigma$ is a small parameter. Through the limit process

$$
\lim _{\sigma \rightarrow 0} \frac{\left[\left.T_{1}^{(1)}\right|_{\lambda=\lambda_{1}+\sigma}\right] \varphi_{2}}{\sigma}=\lim _{\sigma \rightarrow 0} \frac{\left[\sigma+\left.T_{1}^{(1)}\right|_{\lambda=\lambda_{1}}\right] \varphi_{2}}{\sigma}=\varphi_{1}+\left.T_{1}^{(1)}\right|_{\lambda=\lambda_{1}} \varphi_{1}^{[1]} \equiv \varphi_{1}^{(1)},
$$

we can find another solution to the linear system Eq. (6) with $u^{(1)}$ and spectral parameter $\lambda=\lambda_{1}$. This allows us to go to the next step of the DT, namely, Eq. (24).

Similarly, the limit

$$
\begin{aligned}
& \lim _{\sigma \rightarrow 0} \frac{\left[\sigma+\left.T_{1}^{(2)}\right|_{\lambda=\lambda_{1}}\right]\left[\sigma+\left.T_{1}^{(1)}\right|_{\lambda=\lambda_{1}}\right] \varphi_{2}}{\sigma^{2}} \\
& \quad=\varphi_{1}+\left.\left[T_{1}^{(1)}+T_{1}^{(2)}\right]\right|_{\lambda=\lambda_{1}} \varphi_{1}^{[1]}+\left.\left.T_{1}^{(2)}\right|_{\lambda=\lambda_{1}} T_{1}^{(1)}\right|_{\lambda=\lambda_{1}} \varphi_{1}^{[2]} \equiv \varphi_{1}^{(2)}
\end{aligned}
$$

provides a nontrivial solution for the linear spectral problem with $u^{(2)}$ and $\lambda=\lambda_{1}$. Thus, we may do the third-step iteration of the DT as follows:

$$
\varphi^{(3)}=T_{1}^{(3)} \varphi^{(2)}, \quad u^{(3)}=u^{(2)}-\frac{2 i s_{21}^{(2)}}{\delta} .
$$

Continuing this process and combining all the DT, a generalized DT is constructed. Thus, we have the following theorem.

Theorem 2 Let $\varphi_{1}, \varphi_{2}, \ldots, \varphi_{N}$ be $N$ distinct solutions of the spectral problem Eq. (6) at $\lambda_{1}, \ldots, \lambda_{N}$, respectively, and

$$
\varphi_{i}\left(\lambda_{i}+\sigma\right)=\varphi_{i}+\varphi_{i}^{[1]} \sigma+\varphi_{i}^{[2]} \sigma^{2}+\cdots+\varphi_{i}^{[N]} \sigma^{N}+\cdots \quad(i=1,2, \ldots, n)
$$

be their expansions, where

$$
\varphi_{i}^{[j]}=\left.\frac{1}{j !} \frac{\partial^{j}}{\partial \lambda^{j}} \varphi_{i}(\lambda)\right|_{\lambda=\lambda_{i}} \quad(j=1,2, \ldots) .
$$

Define

$$
T=\Gamma_{n} \Gamma_{n-1} \cdots \Gamma_{1} \Gamma_{0}, \quad \Gamma_{k}=T_{k}^{\left(m_{k}\right)} \cdots T_{k}^{(1)} \quad(i \geq 1), \quad \Gamma_{0}=I
$$

where

$$
\begin{aligned}
& T_{k}^{(j+1)}=\left(\begin{array}{cc}
\lambda_{k}-s_{11}^{(j)} & -s_{12}^{(j)} \\
-s_{21}^{(j)} & \left.\lambda_{k}-s_{22}\right)
\end{array}\right), \\
& \varphi_{k}^{(j)}=\lim _{\sigma \rightarrow 0} \frac{\left[\sigma+T_{k}^{(j)} \mid \lambda=\lambda_{k}\right] \cdots\left[\sigma+T_{k}^{(2)} \mid \lambda=\lambda_{k}\right]\left[\sigma+T_{k}^{(1)} \mid \lambda=\lambda_{k}\right] \Gamma_{k-1}\left(\lambda_{k}+\sigma\right) \cdots \Gamma_{1}\left(\lambda_{k}+\sigma\right) \Gamma_{0} \varphi_{k}\left(\lambda_{k}+\sigma\right)}{\sigma^{j}}
\end{aligned}
$$

$\left(1 \leq j<m_{i}\right)$. Then the transformations

$$
\varphi^{(N)}=T \varphi, \quad u^{(N)}=u+\frac{2 i}{\delta} \sum_{j=0}^{n-1} \sum_{k=1}^{m_{i}} s_{21}^{(j)}\left[m_{k}\right] \quad\left(N=n-1+\sum_{k=1}^{n-1} m_{k}\right)
$$

are the generalized DT for the general Hirota equation (4). 
We remark that the solution expressed in Eq. (55) is written in terms of summations, which is very easy to understand. In fact, for nonzero $\varphi^{(k)}(k=1,2, \ldots, N)$, all the denominators of $s_{21}^{(j)}$ are easily seen to be nonzero in these forms; therefore, Eq. (55) provides some nonsingular solutions.

Let us consider an example to illustrate the application of Theorem 2 to the construction of rogue wave solutions. To do so, starting with the seed solution $u=e^{8 i t}$, the solution of the linear spectral problem (6) at $\lambda=i h$ is

$$
\varphi_{1}(f)=\left(\begin{array}{c}
e^{B}\left(C_{1} e^{A}+C_{2} e^{-A}\right) \\
i e^{-B}\left(C_{1} e^{-A}-C_{2} e^{A}\right)
\end{array}\right)
$$

where

$$
C_{1}=\frac{\sqrt{\left(2 \sqrt{\left(4+\lambda^{2}\right)}+2 \lambda\right)}}{2}, \quad C_{2}=\frac{\sqrt{\left(2 \sqrt{\left(4+\lambda^{2}\right)}-2 \lambda\right)}}{2}, \quad B=4 \text { it. }
$$

Let $h=2+f^{2}$. Expanding the vector function $\varphi_{1}(f)$ at $f=0$, we have

$$
\varphi_{1}(f)=\varphi_{1}^{[0]}+\varphi_{1}^{[1]} f^{2}+\varphi_{1}^{[2]} f^{4}+\varphi_{1}^{[3]} f^{6}+\cdots,
$$

where

$$
\begin{aligned}
\varphi_{1}^{[0]} & =\left(\begin{array}{c}
\sqrt{2} e^{4 i t} \\
-\sqrt{2} e^{-4 i t}
\end{array}\right), \\
\varphi_{1}^{[1]} & =\left(\begin{array}{c}
\frac{\sqrt{2}}{200} e^{4 i t}\left((7,680+4,096 i) t^{2}+(800-480 i) t-400 i x^{2}-200 i x+(3,200-1,920 i) x t-25 i\right) \\
-\frac{\sqrt{2}}{200} e^{-4 i t}\left((7,680+4,096 i) t^{2}+(480 i-800) t-400 i x^{2}+200 i x+(3,200-1,920 i) x t-25 i\right)
\end{array}\right), \\
\varphi_{1}^{[2]} & =\left(\begin{array}{c}
P \\
Q
\end{array}\right),
\end{aligned}
$$

with

$$
\begin{aligned}
P= & \frac{\sqrt{2} e^{i t}}{240,000}\left(1,875-456,000 t-30,000 x-1,152,000 x^{2} t+64,880,640 t^{3} x\right. \\
& -1,536,000 t x^{3}+9,830,400 t^{2} x^{2}+4,915,200 x t^{2}-2,400,000 t x+5,836,800 t^{2} \\
& -180,000 x^{2}-160,000 x^{4}-160,000 x^{3}+16,220,160 t^{3}+42,205,184 t^{4} \\
& -1,920,000 i t x^{2}-4,608,000 i t b_{1}+480,000 d_{1}-2,560,000 i x^{3} t+1,920,000 x d_{1} \\
& -3,360,000 i x t-1,920,000 i x b_{1}+7,680,000 t b_{1}+4,608,000 t d_{1}-819,200 i t^{3} \\
& -9,216,000 i t^{2} x-600,000 i t-18,432,000 i t^{2} x^{2}-3,276,800 i t^{3} x \\
& \left.+7,680,000 i t d_{1}-14,208,000 i t^{2}+62,914,560 i t^{4}-480,000 i b_{1}\right) \\
Q= & \frac{\sqrt{2} e^{i t}}{240,000}\left(1,875+456,000 t+30,000 x-1,920,000 i x b_{1}-3,276,800 i t^{3} x\right. \\
& +480,000 i b_{1}+1,152,000 x^{2} t+64,880,640 t^{3} x-1,536,000 t x^{3}+9,830,400 t^{2} x^{2}
\end{aligned}
$$


(a)

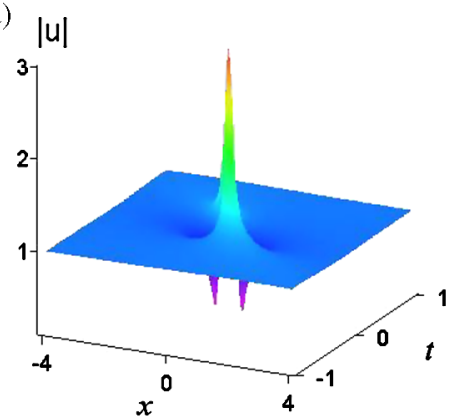

(b)

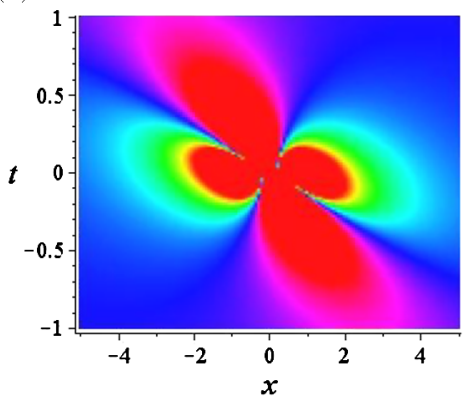

Figure 9 Profile and contour plots of first-order rogue wave. (a) Evolution of the first-order rogue wave solution $u_{r w}^{(1)}$ via (65) with parameters $\alpha=1, \epsilon=0.1, \delta=2, a=0, \lambda=2 i$. (b) The corresponding density plot of $u_{r w}^{(1)}$ in (a).

$$
\begin{aligned}
& -4,915,200 x t^{2}-2,400,000 t x+7,680,000 i t d_{1}-3,360,000 i x t+5,836,800 t^{2} \\
& -180,000 x^{2}-160,000 x^{4}+160,000 x^{3}-16,220,160 t^{3}+42,205,184 t^{4} \\
& -2,560,000 i x^{3} t-4,608,000 i t b_{1}+1,920,000 i t x^{2}+819,200 i t^{3}+1,920,000 x d_{1} \\
& +600,000 i t-14,208,000 i t^{2}+62,914,560 i t^{4}+9,216,000 i t^{2} x \\
& \left.+7,680,000 t b_{1}+4,608,000 t d_{1}-18,432,000 i t^{2} x^{2}-480,000 d_{1}\right)
\end{aligned}
$$

It is clear that $\varphi_{1}^{[0]}$ is a solution for Eq. (6) at $\lambda=2 i$. In the following, we only discuss two cases, $N=1$ and $N=2$.

(1) When $N=1$, by means of formula (47) we can obtain

$$
\varphi_{1}^{(1)}=\lim _{f \rightarrow 0} \frac{\left[f^{2}+T_{1}^{(1)}\right] \varphi_{1}(f)}{f^{2}}=T_{1}^{(1)} \varphi_{1}^{[1]}+i \varphi_{1}^{[0]}
$$

From Eqs. (59), (60), and (64) we derive a simplified form of the first-order rogue wave solution:

$$
u_{r w}^{(1)}=-\frac{e^{8 i t}\left(75-8,704 t^{2}-1,920 x t-400 x^{2}+1,600 i t\right)}{25+8,704 t^{2}+1,920 x t+400 x^{2}},
$$

where $u_{r w}^{(N)}$ is the $N$ th-order rogue wave solution.

Figure 9 displays the evolution and density plots of $u_{r w}^{(1)}$ in Eq. (65), which shows that the first-order rogue wave solution is localized in both space and time.

(2) When $N=2$, by means of formula (54) we have

$$
\varphi_{1}^{(2)}=\lim _{f \rightarrow 0} \frac{\left[f^{2}+T_{1}^{(2)}\right]\left[f^{2}+T_{1}^{(1)}\right] \varphi_{1}(f)}{f^{4}}=T_{1}^{(2)} T_{1}^{(1)} \varphi_{1}^{[2]}+\left(T_{1}^{(1)}+T_{1}^{(2)}\right) \varphi_{1}^{[1]}+\varphi_{1}^{[0]}
$$

which helps to derive the second-order rogue wave solution with parameters $b_{1}=0, d_{1}=0$ in Eqs. (62) and (63) as

$$
u_{r w}^{(2,1)}=\frac{M_{1}}{N_{1}}
$$



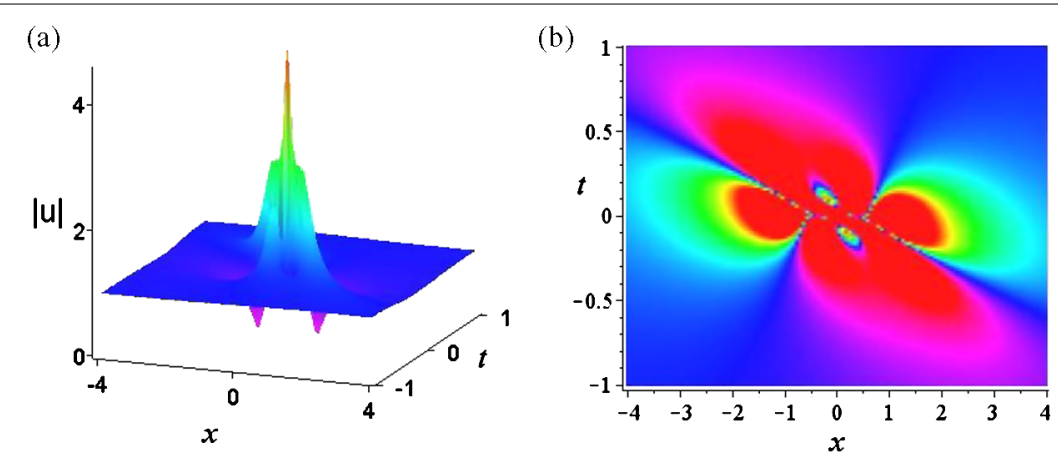

Figure 10 Profile and contour plots of second-order rogue wave of first kind. (a) Evolution of the second-order rogue wave solution $u_{r w}^{(2,1)}$ with parameters $\alpha=1, \epsilon=0.1, \delta=2, a=0, \lambda=2 i$. (b) The corresponding density plot of $u_{r w}^{(2,1)}$ in (a).

with

$$
\begin{aligned}
M_{1}= & e^{8 i t}\left(-659,411,697,664 t^{6}+(363,646,156,800 i-436,375,388,160 x) t^{5}\right. \\
& +\left(160,432,128,000 i x+52,592,640,000-187,170,816,000 x^{2}\right) t^{4} \\
& +\left(4,300,800,000 i-47,185,920,000 x^{3}\right) t^{3}+\left(8,110,080,000 x^{2}\right. \\
& \left.-8,601,600,000 x^{4}+460,800,000 i x+7,372,800,000 i x^{3}+648,000,000\right) t^{2} \\
& +\left(140,400,000 x+499,200,000 x^{3}-45,000,000 i-288,000,000 i x^{2}\right. \\
& \left.\left.-921,600,000 x^{5}\right) t-64,000,000 x^{6}-703,125\right) \\
N_{1}= & 659,411,697,664 t^{6}+436,375,388,160 x t^{5}+\left(187,170,816,000 x^{2}\right. \\
& +36,981,964,800) t^{4}-\left(147,456,000 x-47,185,920,000 x^{3}\right) t^{3} \\
& +\left(596,160,000-1,843,200,000 x^{2}+8,601,600,000 x^{4}\right) t^{2} \\
& +\left(921,600,000 x^{5}-38,400,000 x^{3}+61,200,000 x\right) t \\
& +140,625+64,000,000 x^{6}+12,000,000 x^{4}+6,750,000 x^{2}
\end{aligned}
$$

We show the structure of the second-order rogue wave solution Eq. (67) in Figure 10 under the parameters $\alpha=1, \epsilon=0.1, \delta=2, a=0, \lambda=2 i$.

When choosing parameters $b_{1}=100, d_{1}=0$ in Eqs. (62) and (63), we can obtain another second-order rogue wave solution of the general Hirota equation (4) as

$$
u_{r w}^{(2,2)}=\frac{M_{2}}{N_{2}}
$$

with

$$
\begin{aligned}
M_{2}= & \left(-659,411,697,664 t^{6}+(363,646,156,800 i-436,375,388,160 x) t^{5}\right. \\
& +\left(52,592,640,000-187,170,816,000 x^{2}+160,432,128,000 i x\right) t^{4} \\
& +\left(98,304,000,000+24,920,064,000 x-47,185,920,000 x^{3}\right. \\
& \left.+4,300,800,000 i+51,118,080,000 i x^{2}\right) t^{3}+(648,000,000
\end{aligned}
$$



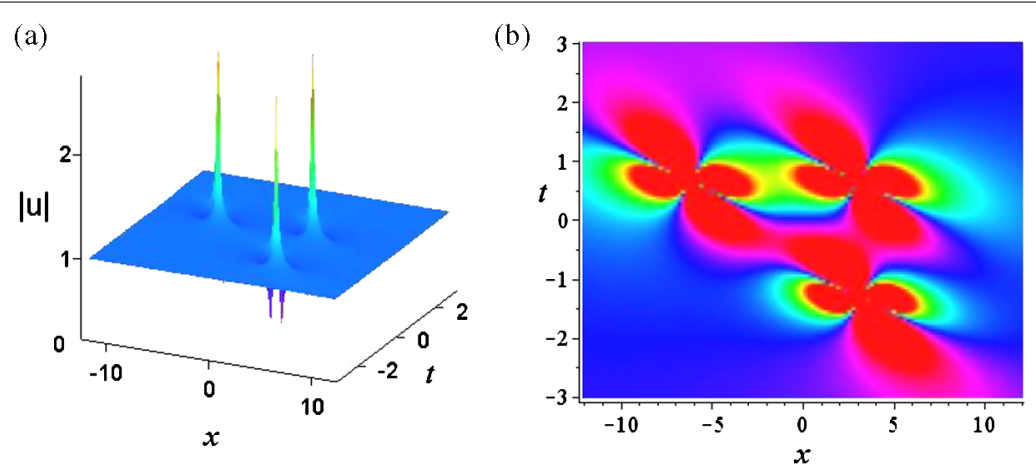

Figure 11 Profile and contour plots of second-order rogue wave of second kind. (a) Evolution plot of the second-order rogue wave solution $u_{r w}^{(2,2)}$ with parameters $\alpha=1, \epsilon=0.1, \delta=2, a=0, \lambda=2 i$. (b) The corresponding density plot of $u_{r w}^{(2,2)}$ in (a).

$$
\begin{aligned}
& +8,110,080,000 x^{2}-8,601,600,000 x^{4}+1,105,920,000,000 x \\
& \left.+294,912,000,000 i+7,372,800,000 i x^{3}+460,800,000 i x\right) t^{2} \\
& +\left(14,400,000,000+140,400,000 x+499,200,000 x^{3}-921,600,000 x^{5}\right. \\
& +230,400,000,000 x^{2}-45,000,000 i+768,000,000 i x^{4} \\
& \left.-138,240,000,000 i x-288,000,000 i x^{2}\right) t+(1,125,000-28,800,000,000 i) x^{2} \\
& +36,000,000 x^{4}-64,000,000 x^{6}-1,440,000,703,125 \\
& -1,800,000,000 i) e^{8 i t} \\
N_{2}= & 659,411,697,664 t^{6}+436,375,388,160 x t^{5}+(36,981,964,800 \\
& \left.+187,170,816,000 x^{2}\right) t^{4}-(98,304,000,000+147,456,000 x \\
& \left.-47,185,920,000 x^{3}\right) t^{3}+(596,160,000-1,105,920,000,000 x \\
& \left.-1,843,200,000 x^{2}+8,601,600,000 x^{4}\right) t^{2} \\
& +\left(921,600,000 x^{5}-230,400,000,000 x^{2}-38,400,000 x^{3}\right. \\
& +61,200,000 x+43,200,000,000) t+12,000,000 x^{4} \\
& +64,000,000 x^{6}+1,440,000,140,625+6,750,000 x^{2}
\end{aligned}
$$

We show the structure of the second-order rogue wave solution in Eq. (70) by Figure 11 under the parameters $\alpha=1, \epsilon=0.1, \delta=2, a=0, \lambda=2 i$.

\section{Conservation laws}

Conservation laws play a key role in discussing the integrability for the nonlinear wave equations. In what follows, we would like to derive the conservation laws of the general Hirota equation (4) by using the linear spectral problem (6), that is,

$$
\begin{aligned}
& \varphi_{1 x}+i \lambda \varphi_{1}=\delta u \varphi_{2}, \quad \varphi_{2 x}-i \lambda \varphi_{2}=-\delta u^{*} \varphi_{1}, \\
& \varphi_{1 t}=A \varphi_{1}+B \varphi_{2}, \quad \varphi_{2 t}=C \varphi_{1}-A \varphi_{2} .
\end{aligned}
$$


Setting $\Gamma_{1}=\frac{\varphi_{2}}{\varphi_{1}}$, we have the following equations from Eqs. (73) and (74):

$$
\begin{aligned}
& \Gamma_{1 x}=2 i \lambda \Gamma_{1}-\delta u^{*}-\delta u \Gamma_{1}^{2}, \\
& \Gamma_{1 t}=C-2 A \Gamma_{1}-B \Gamma_{1}^{2} .
\end{aligned}
$$

From Eqs. (73)-(76) it is easy to get the following equation:

$$
\delta u \Gamma_{1}=\frac{-\delta^{2} u u^{*}-\left(\delta u \Gamma_{1}\right)^{2}-\delta u\left(\frac{\delta u \Gamma_{1}}{\delta u}\right)_{x}}{-2 i \lambda} .
$$

Expanding $\delta u \Gamma_{1}$ into the power series with respect to $(-i \lambda)$,

$$
\delta u \Gamma_{1}=\sum_{n=1}^{\infty} \frac{f_{n}}{(-i \lambda)^{n}},
$$

and substituting Eq. (78) into Eq. (77), we can get the following recursion relations:

$$
\sum_{n=1}^{\infty} \frac{f_{n}}{(-i \lambda)^{n}}=\frac{-\delta^{2} u u^{*}-\left[\sum_{n=1}^{\infty} \frac{f_{n}}{(-i \lambda)^{n}}\right]^{2}-\delta u\left[\sum_{n=1}^{\infty} \frac{f_{n}}{(-i \lambda)^{n} \delta u}\right]_{x}}{-2 i \lambda} .
$$

Furthermore, the following conservation law equation can be obtained from Eqs. (73)(76):

$$
\left(\delta u \Gamma_{1}\right)_{t}=\left(A+B \Gamma_{1}\right)_{x} .
$$

Substituting Eq. (79) into Eq. (80), we derive an infinite number of conservation laws for Eq. (4) immediately. The first three conservation laws describe the energy conservation, momentum conservation, and Hamiltonian conservation. We list them as follows:

$$
\begin{aligned}
\left(u u^{*}\right)_{t}= & {\left[3 \delta^{2} \epsilon u^{2} u^{* 2}+\epsilon\left(u u_{x x}^{*}-u_{x} u_{x}^{*}+u_{x x} u^{*}\right)-i \alpha\left(u u_{x}^{*}-u_{x} u^{*}\right)\right]_{x} } \\
\left(u u_{x}^{*}\right)_{t}= & {\left[4 \delta^{2} \epsilon u^{2} u^{*} u_{x}^{*}+\epsilon\left(u u_{x x x}^{*}-u_{x} u_{x x}^{*}+2 u_{x x} u_{x}^{*}+4 u^{2} u^{*} u_{x}^{*}\right)\right.} \\
& \left.\quad \delta^{2} i \alpha u^{2} u^{* 2}+i \alpha\left(2 u_{x} u_{x}^{*}-u u_{x x}^{*}\right)\right]_{x}, \\
\left(u^{2} u^{*}+u u_{x x}^{*}\right)_{t} & \\
= & {\left[\delta^{2} \alpha_{3}\left(8 u^{2} u^{*} u_{x x}^{*}+5 u^{2} u_{x}^{* 2}+2 u u_{x} u^{*} u_{x}^{*}+u u_{x x} u^{* 2}+u u_{x x x x}^{*}-u_{x}^{2} u^{* 2}+u u^{* 2} u_{x x}\right)\right.} \\
+ & \left.4 \delta^{4} \epsilon u^{3} u^{* 3}-4 \delta^{2} i \alpha u^{2} u^{*} u_{x}^{*}+i \alpha\left(u_{x} u_{x x}^{*}-u u_{x x x}^{*}\right)+\epsilon\left(u_{x x} u_{x x}^{*}-u_{x} u_{x x x}^{*}\right)\right]_{x} .
\end{aligned}
$$

\section{Conclusions}

In summary, we have studied the exact solutions and conservation laws of the general Hirota equation (4) based on its linear spectral problem (6). We have derived an $N$-fold Darboux transformation of this equation and then obtained some exact multisoliton solutions and breather solutions. Introducing a generalized Darboux transformation, some rogue wave solutions of this equation have been also proposed explicitly. Moreover, the dynamical features of these exact solutions are analyzed by their density distributions. At last, the conservation laws of this general Hirota equation are proposed by means of its linear spectral problem. 


\section{Competing interests}

The authors declare that they have no competing interests.

\section{Authors' contributions}

All authors have made equal contributions. All authors have read and approved the final manuscript.

\section{Acknowledgements}

This work is supported by National Natural Science Foundation of China under Grant Nos. 11271362 and 11375030, Beijing Natural Science Foundation under Grant No. 1153004, Beijing Natural Science Fund Project and Beijing City Board of Education Science and Technology Key Project No. KZ201511232034, Beijing Nova program No. Z131109000413029, and Beijing Finance Funds of Natural Science Program for Excellent Talents No. $2014000026833 Z$ Z19.

Received: 3 September 2015 Accepted: 7 February 2016 Published online: 03 March 2016

\section{References}

1. Hasegawa, A, Tappert, F: Transmission of stationary nonlinear optical physics in dispersive dielectric fibers I: anomalous dispersion. Appl. Phys. Lett. 23, 142-144 (1973)

2. Mollenauer, LF, Stolen, RH, Gordon, JP: Experimental observation of picosecond pulse narrowing and solitons in optical fibers. Phys. Rev. Lett. 45, 1095-1098 (1980)

3. Ablowitz, MJ, Clarkson, P: Solitons, Nonlinear Evolution Equations and Inverse Scattering. Cambridge University Press, Cambridge (1992)

4. Ablowitz, MJ, Prinari, B, Trubatch, AD: Discrete and Continuous Nonlinear Schrödinger Systems. Cambridge University Press, Cambridge (2003)

5. Kodama, YJ, Hasegawa, A: Solitons in Optical Communications. Oxford University Press, Oxford (1995)

6. Agrawal, GP: Applications of Nonlinear Fiber Optics. Academic Press, San Diego (2001)

7. Maimistov, Al, Basharov, AM: Nonlinear Optical Waves. Kluwer Academic, Dordrecht (1999)

8. Guo, R, Hao, HQ: Breathers and multi-soliton solutions for the higher-order generalized nonlinear Schrödinger equation. Commun. Nonlinear Sci. Numer. Simul. 18, 2426-2435 (2013)

9. Wang, DS: Optical solitons in a nonlinear fiber medium with higher-order effects. In: Yasin, M, Harun, SW, Arof, H (eds.) Recent Progress in Optical Fiber Research, pp. 49-64 (2012)

10. Herbst, BM, Ablowitz, MJ, Ryan, E: Numerical homoclinic instabilities and the complex modified Korteweg-de Vries equation. Comput. Phys. Commun. 65, 137-142 (1991)

11. Ankiewicz, A, Soto-Crespo, JM, Akhmediev, N: Rogue waves and rational solutions of the Hirota equation. Phys. Rev. E $81,046602(2010)$

12. Tao, Y, He, J: Multisolitons, breathers and rogue waves for the Hirota equation generated by the Darboux transformation. Phys. Rev. E 85, 026601 (2012)

13. Chowdury, A, Ankiewicz, A, Akhmediev, N: Moving breathers and breather to soliton conversions for the Hirota equation. Proc. R. Soc. A 471, 20150130 (2015)

14. Baleanu, D, Killic, B, Ugurlu, Y, Inc, M: The first integral method for the (3+1)-dimensional modified Korteweg-de Vries-Zakharov-Kuznetsov and Hirota equations. Rom. J. Phys. 60, 111-125 (2015)

15. Baleanu, D, Kilic, B, Inc, M: The first integral method for Wu-Zhang nonlinear system with time-dependent coefficients. Proc. Rom. Acad., Ser. A : Math. Phys. Tech. Sci. Inf. Sci. 16, 160-167 (2015)

16. Akgul, A, Inc, M, Karatas, E, Baleanu, D: Numerical solutions of fractional differential equations of Lane-Emden type by an accurate technique. Adv. Differ. Equ. 2015, 220 (2015)

17. Meng, XH: Wronskian and Grammian determinant structure solutions for a variable-coefficient forced Kadomtsev-Petviashvili equation in fluid dynamics. Physica A 413, 635-642 (2014)

18. Al-Harbi, WG: Numerical solution of Hirota equation. Master's thesis, Umm Al-Qura University (2009)

19. Gu, CH, He, HS, Zhou, ZX: Darboux Transformation in Soliton Theory and Its Geometric Applications. Shanghai Scientific and Technical Publishers, Shanghai (2005)

20. Guo, BL, Ling, LM, Liu, QP: Nonlinear Schrödinger equation: generalized Darboux transformation and rogue wave solutions. Phys. Rev. E 85, 026607 (2012)

\section{Submit your manuscript to a SpringerOpen ${ }^{\ominus}$ journal and benefit from:}

- Convenient online submission

Rigorous peer review

- Immediate publication on acceptance

- Open access: articles freely available online

- High visibility within the field

- Retaining the copyright to your article 